\title{
Additive Manufacturing for Solid Oxide Cell Electrode Fabrication
}

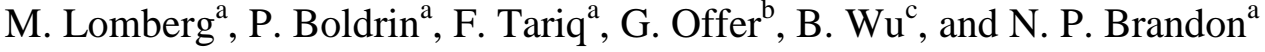 \\ ${ }^{a}$ Department of Earth Science and Engineering, Imperial College London, Exhibition Rd, \\ London SW7 2AZ, UK \\ ${ }^{\mathrm{b}}$ Department of Mechanical Engineering, Imperial College London, Exhibition Rd, \\ London SW7 2AZ, UK \\ ${ }^{c}$ Dyson School of Design Engineering, Imperial College London, Exhibition Rd, London
} SW7 2AZ, UK

\begin{abstract}
Additive manufacturing can potentially offer a highly-defined electrode microstructure, as well as fast and reproducible electrode fabrication. Selective laser sintering is an additive manufacturing technique in which three-dimensional structures are created by bonding subsequent layers of powder using a laser.

Although selective laser sintering can be applied to a wide range of materials, including metals and ceramics, the scientific and technical aspects of the manufacturing parameters and their impact on microstructural evolution during the process are not well understood.

In the present study, a novel approach for electrode fabrication using selective laser sintering was evaluated by conducting a proof of concept study. A Ni-patterned fuel electrode was laser sintered on an yttria-stabilized zirconia substrate. The optimization process of laser parameters (laser sintering rate and laser power) and the electrochemical results of a full cell with a laser sintered electrode are presented. The challenges and prospects of using selective laser sintering for solid oxide cell fabrication are discussed.
\end{abstract}

\section{Introduction}

Solid oxide fuel cells (SOFCs) are high temperature fuel cells that operate with a ceramic oxygen-ion conducting electrolyte, such as yttria-stabilized zirconia (YSZ). They typically operate in a temperature range of $500{ }^{\circ} \mathrm{C}-1000^{\circ} \mathrm{C}$ with cermet based anodes and cathodes such as Nickel(Ni)-YSZ and lanthanum strontium cobalt ferrite (LSCF) respectively (1).

The microstructure of the electrodes in solid oxide cells (SOCs) plays a crucial role in determining the electrochemical performance. Greene et al. (2) developed a computational model to show that functionally graded electrodes in SOFCs can improve the performance relative to electrodes with a single bulk material porosity. They show that performance benefits are realised given an increase in porosity near the electrolyte interface.

$\mathrm{Ni}$ et al. (3) later showed through computational modelling, that microstructural grading could significantly enhance gas transport whilst having negligible effect on 
ohmic and activation overpotentials, especially so for thick electrodes. The limitation is for thin electrodes with larger particles, where high grading could result in larger activation overpotentials at low/medium current densities. It was also shown that particle size grading is generally more effective than porosity grading, resulting in a one order of magnitude increase in power density.

Additive manufacturing (AM) techniques, such as selective laser sintering (SLS) and ink-jet printing, are potential fabrication routes to highly defined and hierarchical microstructures with good dimensional tolerance, that can be produced in a fast and controllable way (4). The advantages and opportunities offered by the emerging field of AM are now widely acknowledged, though the number of studies regarding its application in processing SOC-relevant materials is still limited. The scientific and technical aspects of the production route and the effects of manufacturing parameters on the microstructural evolution during the process have not been well explored. Moreover, the numerous combinations of different parameters make this method very complex. As a consequence, this technique currently relies upon experimental data (5).

Bertrand et al. (6) produced various components from yttria-zirconia powders. A porous structure of $56 \%$ density was fabricated. This would be sufficient for use in YSZbased electrodes. The authors highlight the challenge of manufacturing a powder with appropriate characteristics suitable for SLS of $\mathrm{ZrO}_{2}-\mathrm{Y}_{2} \mathrm{O}_{3}$ components.

$\mathrm{La}_{2} \mathrm{Mo}_{2} \mathrm{O}_{9}$-based ceramics can be used as electrolyte materials in SOCs. Yu et al (7) studied electrical conductivity of a dense mixed conducting $\mathrm{La}_{2} \mathrm{Mo}_{1.4} \mathrm{~W}_{0.6} \mathrm{O}_{9}$ ceramic prepared by laser rapid solidification method with a $\mathrm{CO}_{2}$ laser. During fabrication, the ceramic solidifies mainly against the direction of heat transfer producing elongated blocks of over $100 \mu \mathrm{m}$ length. The overall conductivity of the ceramic exhibited strong dependence on the measurement orientation, with higher conductivity measured parallel to the elongated blocks. The authors state that the enhanced electrical conductivity was due to reduced grain boundary resistances in the direction parallel to crystal solidification orientation.

In the same manner, a laser rapid solidification method was used by Zhang el al (8) for the synthesis of $\mathrm{La}_{0.9} \mathrm{Sr}_{0.1} \mathrm{Ga}_{0.8} \mathrm{Mg}_{0.2} \mathrm{O}_{3-\delta}$ (LSGM), an electrolyte for SOFCs. The conductivities of $0.027,0.079$ and $0.134 \mathrm{~S} \mathrm{~cm}^{-1}$ obtained at 600,700 and $800{ }^{\circ} \mathrm{C}$, respectively, are higher than those of the samples fabricated by conventional methods. The optimum conditions for LSGM fabrication were around 1000-1100 W laser power and $1 \mathrm{~mm} \mathrm{~s}^{-1}$ scan speed. The materials solidified in densely packed and relatively ordered ridge-like blocks with a relative density of $98.5 \%$.

In this study the use of SLS for electrode fabrication was evaluated by conducting a proof of concept study. A Ni-patterned fuel electrode was formed on an YSZ substrate by means of laser sintering. The linear laser energy is essentially defined as a ratio between laser power and laser speed. The optimization process of laser parameters (laser speed and laser power) and results of the electrochemical test of a full cell with laser sintered electrode are presented. The challenges and prospects of using SLS for SOC fabrication are discussed. 


\section{Experimental}

Optimization of laser parameters for electrode fabrication

Design of experiments. The laser variable parameters controlled in this study were laser speed $(S)$ and laser power $(P)$, whilst others, such as hatch spacing, laser diameter, were kept unchanged. The experiments were designed so that different settings of laser parameters could be tested with a limited number of samples. To allow this, 5 subsamples with a diameter of $4 \mathrm{~mm}$ were fabricated on each YSZ pellet (of 250-300 $\mu \mathrm{m}$ thickness and $20 \mathrm{~mm}$ diameter) as shown in Figure 1a. The diameters of the fabricated sub-samples are to scale relative to the YSZ substrate. Each pellet and sub-sample was labelled so that the laser parameters could be correlated with the results of the sintering (Figure 1a). Different combinations of laser power and laser speed were applied on each individual sub-sample. However, the energy density, defined as the ratio between laser power and laser speed, on each sub-sample within the same pellet was kept constant on all samples. The energy density varied between pellets.

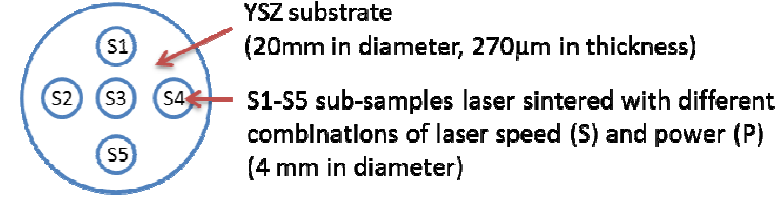

(a)

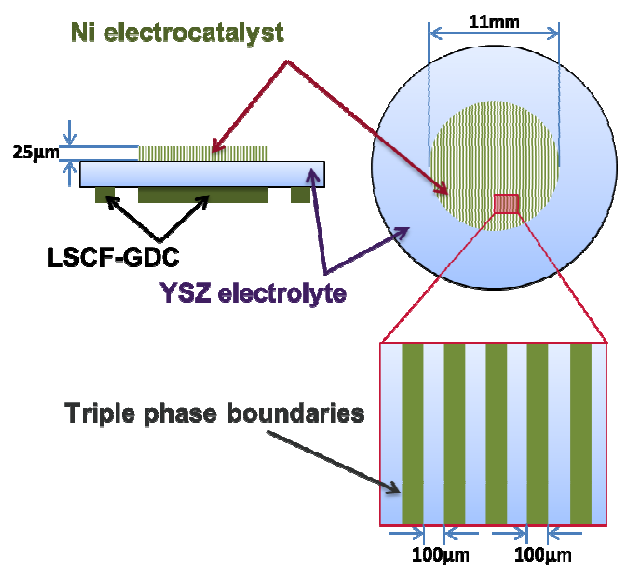

Figure 1. (a) Diagram of the YSZ pellet (substrate) carrying 5 sub-samples (S1-S5) to be laser sintered, the diameters of the sub-samples are to scale relative to the YSZ substrate. (b) Diagram of the YSZ pellet with screen-printed LSCF-GDC air and reference electrodes on one side and patterned laser sintered Ni working electrode on the other side.

Samples fabrication by Selective Laser Sintering. To find an optimum combination of laser parameters, a wide spectra of laser speeds (in the range of $200-6000 \mathrm{~mm} / \mathrm{sec}$ ) and laser power (in the range of $20-190 \mathrm{~W}$ ) were used to fabricate samples. An EOSINT M 270 with a $200 \mathrm{~W}$ YAG pumped fibre pulse laser $(\lambda=1.06 \mu \mathrm{m}$, laser frequency $8000 \mathrm{~Hz}$, laser spot size $100 \mu \mathrm{m}$ ) in a $98.5 \% \mathrm{~N}_{2}-1.5 \% \mathrm{O}_{2}$ atmosphere was used for 3D laser sintering. The pellet holder was custom made from stainless steel to hold the YSZ substrates. During the SLS experiments, the holder was heated to $80{ }^{\circ} \mathrm{C}$. Ni powder (5-15 $\mu \mathrm{m}$ diameter spherical particles) was used to create patterned laser sintered structures. Each layer thickness was $20 \mu \mathrm{m}$ and up to 3 layers were sintered. 
$\underline{\text { Full cell preparation, testing and characterization }}$

A full fuel cell with patterned laser sintered anode was fabricated and tested to obtain initial electrochemical results. For this purpose the LSCF-GDC counter $(11 \mathrm{~mm}$ in diameter) and reference (16 and $18 \mathrm{~mm}$ inner and outer diameters) electrodes were first screen printed on the YSZ electrolyte as described elsewhere (9). Then the patterned electrode $(11 \mathrm{~mm}$ in diameter) was laser sintered on the other side of the substrate, as illustrated in Figure 1b. The choice of laser parameters combination used for the patterned $\mathrm{Ni}$ electrode fabrication was based on the previous steps, in which the optimized laser conditions were conceived, as will be discussed further.

The electrochemical performance of Ni patterned electrodes, laser sintered on an YSZ electrolyte, was tested. Impedance spectra $(100 \mathrm{mHz}-100 \mathrm{kHz}$, with an AC amplitude of $6 \mathrm{~mA}$ ) and $i-V$ curves were measured in three electrode set-ups as was explained elsewhere (9) in a humidified $50 \% \mathrm{H}_{2}-50 \% \mathrm{~N}_{2}$ atmosphere at $600-700{ }^{\circ} \mathrm{C}$. Current collection was provided through silver paste.

The microstructure was examined using a scanning electron microscope (SEM) JSM 6010 LA microscope fitted with Oxford instruments INCA energy dispersive x-ray spectrometer (EDX). The EDX data was accumulated from 20 sweeps with $0.1 \mathrm{~ms}$ point dwell time. The images were taken in a secondary electrons mode; the accelerating voltage of $20 \mathrm{kV}$ was used. Prior to microstructure analysis the samples were coated with chromium (ca. $30 \mathrm{~nm}$ ).

\section{Results and Discussion}

Optimization of laser parameters for electrode fabrication

Table I outlines the matrix of sample assignments. In Table I each row (R1-R3) indicates the number of layers fabricated and each column corresponds to the energy density applied on each pellet in that column $(0.03-0.15 \mathrm{~J} / \mathrm{mm})$.

Table I. Matrix of substrates assignments (YSZ pellets) as arranged in the SLS machine chamber.

\begin{tabular}{|c|c|c|c|c|c|c|}
\hline \multirow{2}{*}{$\begin{array}{l}\text { Row } \\
\text { number }\end{array}$} & \multirow{2}{*}{$\begin{array}{l}\text { Number of layers } \\
\text { (layer thickness }=20 \mu \mathrm{m})\end{array}$} & \multicolumn{5}{|c|}{ (Lateral) Energy Density [Wsec/mm=J/mm] } \\
\hline & & 0.03 & 0.05 & 0.07 & 0.1 & 0.15 \\
\hline R1 & $\mathrm{L}=1$ & 1.1 & 1.2 & 1.3 & 1.4 & 1.5 \\
\hline R2 & $\mathrm{L}=2$ & 2.1 & 2.2 & 2.3 & 2.4 & 2.5 \\
\hline $\mathbf{R 3}$ & $\mathrm{L}=3$ & 3.1 & 3.2 & 3.3 & 3.4 & 3.5 \\
\hline
\end{tabular}

Table II summarizes the laser parameters applied for each individual sub-sample (S1-S5) within the samples (1.1-3.5) listed in Table I. In total 5 × 5 × $3=75$ sub-samples were fabricated in the same run.

All samples fabricated with an energy density $>0.05 \mathrm{~J} / \mathrm{mm}$ fractured after one layer was sintered, independently of the combination of laser power and laser speed. Among samples prepared with the laser energy of $0.05 \mathrm{~J} / \mathrm{mm}$, only Sample 1.2 (one sintered layer) did not fracture and the others, with two or three layers, failed. In contrast, none of the samples fabricated with $0.03 \mathrm{~J} / \mathrm{mm}$ fractured, even after three laser sintered layers. 
Table II. Summary of the laser parameters used for each sub-sample.

\begin{tabular}{|c|c|c|c|c|c|c|c|c|}
\hline \multicolumn{9}{|c|}{ (Lateral) Energy Density [Wsec/mm=J/mm] } \\
\hline \multicolumn{3}{|c|}{0.03} & \multicolumn{3}{|c|}{0.05} & \multicolumn{3}{|c|}{0.07} \\
\hline $\begin{array}{c}\text { Samples } \\
\text { 1.1-3.1 }\end{array}$ & $\begin{array}{c}\mathrm{LS} \\
\mathrm{mm} / \mathrm{sec}\end{array}$ & $\begin{array}{l}\text { LP, } \\
\text { W }\end{array}$ & $\begin{array}{c}\text { Samples } \\
1.2-3.2\end{array}$ & $\begin{array}{c}\mathrm{LS} \\
\mathrm{mm} / \mathrm{sec}\end{array}$ & $\begin{array}{c}\mathbf{L P}, \\
\mathbf{W}\end{array}$ & $\begin{array}{c}\text { Samples } \\
\text { 1.3-3.3 }\end{array}$ & $\begin{array}{c}\mathrm{LS} \\
\mathrm{mm} / \mathrm{sec}\end{array}$ & $\begin{array}{l}\text { LP, } \\
\text { W }\end{array}$ \\
\hline S1 & 1333 & 40 & S1 & 800 & 40 & S1 & 571 & 40 \\
\hline $\mathbf{S 2}$ & 2000 & 60 & $\mathbf{S 2}$ & 1600 & 80 & $\mathbf{S 2}$ & 1143 & 80 \\
\hline S3 & 2667 & 80 & S3 & 2000 & 100 & S3 & 1430 & 100 \\
\hline S4 & 3333 & 100 & S4 & 2800 & 140 & S4 & 2000 & 140 \\
\hline S5 & 4000 & 120 & S5 & 3800 & 190 & S5 & 2715 & 190 \\
\hline \multicolumn{9}{|c|}{ (Lateral) Energy Density [Wsec/mm=J/mm] } \\
\hline \multicolumn{3}{|c|}{0.1} & \multicolumn{3}{|c|}{0.15} & Other det: & & \\
\hline $\begin{array}{c}\text { Samples } \\
1.4-3.4\end{array}$ & $\begin{array}{l}\mathrm{LS} \\
\mathrm{mm} / \mathrm{sec}\end{array}$ & $\begin{array}{l}\text { LP, } \\
\text { W }\end{array}$ & $\begin{array}{c}\text { Samples } \\
1.5-3.5\end{array}$ & $\begin{array}{l}\mathrm{LS} \\
\mathrm{mm} / \mathrm{sec}\end{array}$ & $\begin{array}{l}\text { LP, } \\
\text { W }\end{array}$ & \multirow{6}{*}{\multicolumn{3}{|c|}{$\begin{array}{l}\text { Laser parameters for contour: } \\
100 \mathrm{~W} 3333 \mathrm{~mm} / \mathrm{sec} \text { before and } \\
\text { after scan }(0.03 \mathrm{~J} / \mathrm{mm})\end{array}$}} \\
\hline S1 & 200 & 20 & S1 & 133 & 20 & & & \\
\hline $\mathbf{S 2}$ & 400 & 40 & $\mathbf{S 2}$ & 267 & 40 & & & \\
\hline S3 & 800 & 80 & S3 & 533 & 80 & & & \\
\hline S4 & 1400 & 140 & S4 & 933 & 140 & & & \\
\hline S5 & 1900 & 190 & S5 & 1267 & 190 & & & \\
\hline
\end{tabular}

Between the two consecutive layers there was a ca.1 minute pause to allow spreading of the next powder layer. Two possible explanations can be put forward to explain why repetitive laser sintering caused the substrate to break.

- It is likely that the damage to the substrate is proportional to the energy density. Hence, the results might suggest that in samples 2.2-3.2, fabricated with higher energy density $(0.05 \mathrm{~J} / \mathrm{mm})$, the accumulation of thermal stresses after applying repetitive laser energy exceed the critical value for crack propagation and caused substrate failure.

- Furthermore, given that the volume of melted Ni increases with laser energy density, upon melting and cooling bigger particles of Ni could cause higher stresses on the YSZ substrate, due to the thermal expansion coefficient mismatch between $\mathrm{Ni}$ and YSZ $\left(13.3 \times 10^{-6}\right.$ and $8 \times 10^{-6} \mathrm{~K}^{-1}$, respectively). Higher stresses associated with differences in thermal expansion coefficients could result in micro-cracks, which eventually may exceed the critical length for crack propagation and could induce the failure.

It is thus likely that there is a trade-off between sufficient laser power (that causes the powder to partially or fully melt), on one hand, and an energy density limit (above which there are significant thermal stresses affecting bulk integrity), on the other.

\section{Electrochemical performance of laser sintered patterned electrode}

The open circuit potential (OCP) was close to its theoretical value during the cell tests at the considered temperatures. This is a good indication that no macro-cracking of the electrolyte had occurred, which otherwise would have led to a decrease in OCP due to gas crossover.

The $i$ - $V$ curves of the laser sintered patterned electrode (Sample 2.2 from Table I), normalized to the unit of the theoretical TPB length, are shown in Figure 2a. The curves 
were measured in three electrode configurations, i.e. between working (WE) and reference electrodes (RE).

Figure $2 \mathrm{~b}$ shows the impedance spectra of the patterned $\mathrm{Ni}$ electrode measured in three electrode configurations (i.e. between WE and RE). The impedance spectra were normalized to the unit of theoretical TPB length in a similar way to other studies of patterned electrodes (10). The value of the series resistance was normalized to zero to compensate for the variable uncompensated $i R$ drop between the RE and WE under different conditions.
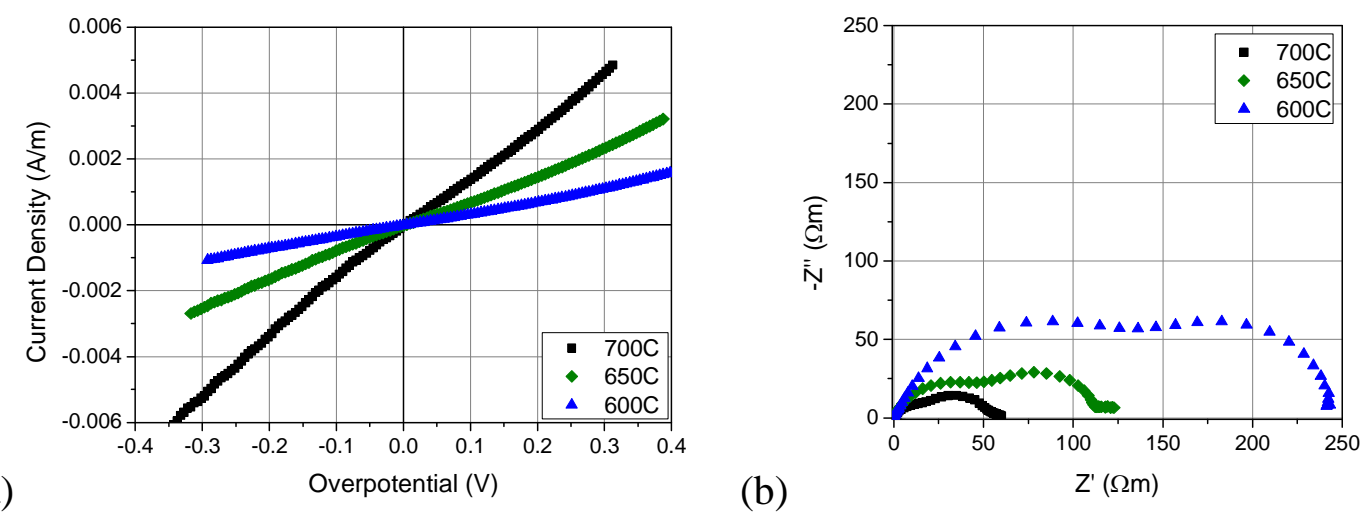

Figure 2. (a) Current linear density (per unit of theoretical length of TPB) vs overpotential of the cell with laser sintered patterned electrode (Sample 2.2), as measured between the RE and WE (the voltage drop includes the contributions from the laser sintered electrode and the uncompensated $i R$ drop between the RE and WE) and (b) Galvanostatic impedance spectra $(100 \mathrm{mHz}-100 \mathrm{kHz})$ of the laser sintered patterned $\mathrm{Ni}$ electrode normalised to the unit of theoretical TPB length on YSZ substrate. Conditions for electrode fabrication are: energy density $=0.03 \mathrm{~J} / \mathrm{mm}$, comprised of laser speed $=$ $4000 \mathrm{~mm} / \mathrm{sec}$ and laser power $=120 \mathrm{~W}$. The cell was tested in humidified $50 \% \mathrm{H}_{2}-50 \%$ $\mathrm{N}_{2}$ under OCP in a $600-700{ }^{\circ} \mathrm{C}$ temperature range.

The total theoretical length of triple phase boundaries (TPBs) of the patterned electrode was estimated, assuming that the interface line between YSZ and Ni is perfectly straight and continuous. A schematic representation of the patterned electrode is shown in Figure $1 \mathrm{~b}$. The width of each stripe is $100 \mu \mathrm{m}$ and the distance between two neighbouring stripes (i.e. hatch spacing) is $100 \mu \mathrm{m}$, the diameter of the entire electrode is $11 \mathrm{~mm}$. Based on these dimensions, the total theoretical length of the TPB was calculated to be $0.96 \mathrm{~m}$ and the TPB length per unit area is therefore $1.01 \times 10^{4} \mathrm{~m} / \mathrm{m}^{2}$. The TPB density was of the same order of magnitude as that previously reported in patterned electrodes $\left(8.7 \times 10^{4} \mathrm{~m} / \mathrm{m}^{2}\right)(10)$.

However, the actual length of the TPB in our electrodes is likely to be larger than the theoretical value due to micro-scale roughness of the Ni/YSZ interface, as seen in the SEM-EDX analysis (Figure 3). On the other hand, agglomeration of Ni during laser sintering resulted in a network of Ni micro-particles that are not interconnected, rather than a solid pattern as shown in Figure 1b. A very low current density in Figure $2 \mathrm{a}$ suggests that only a small part of the electrode is active. 
$\underline{\text { Microstructure characterization of laser sintered electrodes }}$

Figure 3a-c demonstrates SEM micrographs of the Ni patterned electrode surface corresponding to Sample 2.2. The sample was laser sintered under $0.03 \mathrm{~J} / \mathrm{mm}$ (comprised of laser power $120 \mathrm{~W}$ and laser speed $4000 \mathrm{~mm} / \mathrm{sec}$ ).

(a)
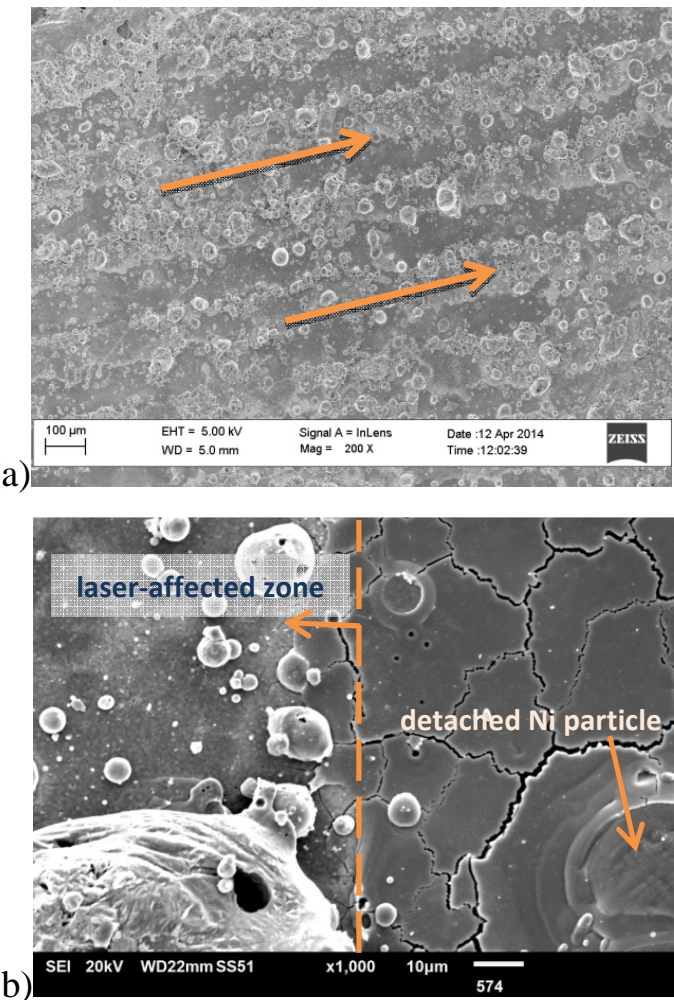

(c)

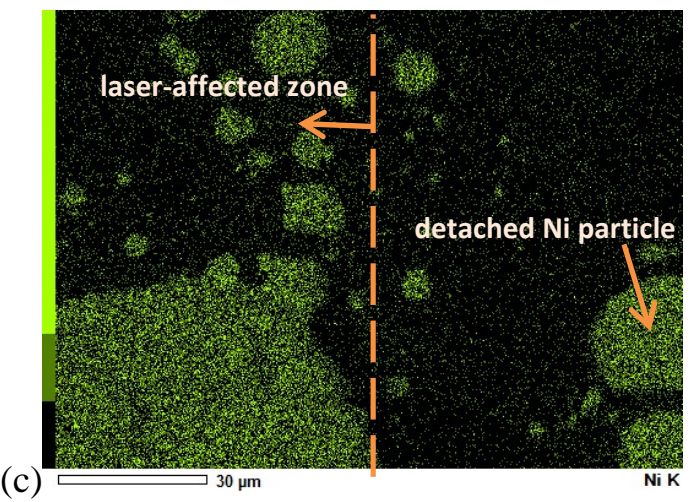

Figure 3. (a) Laser sintered Ni pattern on YSZ surface (Sample 2.2), the arrows identify the direction of laser, (b) zoom in Sample 2.2 surface and (c) SEM-EDX map of Ni distribution (green).

The pattern of about $100 \mu \mathrm{m}$ wide sintered stripes can clearly be seen on the SEM image (Figure 3a), however the edge of the stripes and their surface is not homogeneous. The TPB lines in Figure 3a appear to have an irregular shape, compared to the shape of patterned Ni electrodes on YSZ obtained elsewhere (10-13). Therefore it is difficult to make a direct comparison between the performance of patterned electrodes obtained in this work and in the literature. The lack of connectivity between Ni particles along the laser affected zone may account for the low number of active sites and the low performance of the electrode as shown in Figure 2. One possible route to enhance the adhesion between YSZ and Ni may be metallization of the YSZ surface with a thin sputtered Ni layer prior to laser sintering of Ni powder. This requires further research.

It can also be seen from Figure 3a-b, that the pattern includes multiple separated Ni particles forming 'balls' of up to $50 \mu \mathrm{m}$ in diameter. Balling is a well-known phenomenon associated with the SLS process (14-18). Balling occurs when the molten material does not wet the underlying substrate. Kruth et al (17) experimentally identified a process window for laser sintering iron-based powder. It suggests that the 'balling' phenomenon occurs at low laser speeds and high laser powers. 
Detached Ni particles were also found in the surface that was not affected by laser heat (Figure 3c). This can be as a result of vaporization (19). During laser sintering the temperature of the exposed powder particles exceeds the melting point $\left(1453{ }^{\circ} \mathrm{C}\right.$ for $\left.\mathrm{Ni}\right)$ under high power densities. A further increase of the temperature can cause the material to evaporate. The rapidly moving evaporated powder particles expand and generate a recoil pressure on the molten pool. Under high pressures material expulsion occurs, which may lead to the anchoring of detached particles at some distance from the molten pool (17).

Micro-cracks on the YSZ surface were detected in all samples, independently of laser parameters. It is likely that cracking in YSZ occurs due to a combination of thermal shock and the mismatch between the thermal expansion coefficients of YSZ and $\mathrm{Ni}$ as discussed above.

\section{Conclusions}

In the present study, a novel approach for SOC electrode fabrication using SLS was evaluated in a proof of concept study. A Ni-patterned fuel electrode was laser sintered on an YSZ substrate. Laser energy densities of $0.05 \mathrm{~J} / \mathrm{mm}$ and higher caused the YSZ substrate to break, when attempting to laser sinter more than one layer. Two failure mechanisms were suggested. First, thermal stresses accumulate in each run, which could induce failure. Second, the increasing mismatch in thermal expansion coefficients between YSZ and Ni due to Ni particle growth with every additional sintered layer could further accelerate the cracking. These two mechanisms could both take place at the same time. The results suggest that the maximum laser energy of $0.03 \mathrm{~J} / \mathrm{mm}$, comprised of 120 $\mathrm{W}$ laser power and $4000 \mathrm{~mm} / \mathrm{sec}$ laser speed, seem to be acceptable parameters for cell fabrication, at least as up to three laser sintered Ni layers are concerned. In general, a trade-off should be maintained between sufficient laser power (that causes the powder to partially or fully melt) and laser energy density limit (above which significant thermal stresses accumulation affects bulk integrity).

From microstructural examination it can be concluded that the patterned electrode is prone to a balling phenomenon. Poor wettability can account for the balling phenomenon in this particular system. In general the wettability can be improved by small additions of materials that reduce surface energy (such as phosphorous). In our case the selection of materials would be dictated by the application. One possible route to increase bonding between YSZ and Ni powder may be pre-metallization of the YSZ surface with a thin sputtered layer. This requires further investigation.

The full cells with patterned Ni fuel electrode (laser sintered with $120 \mathrm{~W}$ laser power and $4000 \mathrm{~mm} / \mathrm{sec}$ laser speed), YSZ electrolyte and LSCF-GDC air electrode were successfully fabricated and tested in a $\mathrm{H}_{2}-\mathrm{N}_{2}$ environment at $600-700{ }^{\circ} \mathrm{C}$. Although there was no crossover between the fuel and the air electrode during the experiment, the fact that micro-cracks were introduced to the substrate surface questions the durability of the cell in a long-term. The micro-cracks are likely to originate from thermal shock and mismatch between thermal expansion coefficients of YSZ and Ni.

Fabrication of SOC electrodes using laser sintering is potentially a promising technique to create highly defined hierarchical porous microstructures. The present study highlights the difficulties associated with SLS for SOC electrode fabrication that remain 
to be overcome, in particular materials selection and laser parameter optimization to mitigate micro-cracking and balling phenomena, and to maximize wettability and compatibility between materials.

\section{Acknowledgments}

The authors would like to acknowledge the Alan Howard Scholarship for funding this work, and the EPSRC SUPERGEN Fuel Cell Programme (EP/J016454/1).

\section{References}

1. Subhash C Singhal and K. Kendall, High Temperature Solid Oxide Fuel Cells: Fundamentals, Design and Applications, Elsevier (2003).

2. E. S. Greene, W. K. S. Chiu and M. G. Medeiros, Journal of Power Sources, 161, 225 (2006).

3. M. Ni, M. K. H. Leung and D. Y. C. Leung, Journal of Power Sources, 168, 369 (2007).

4. B. Qian and Z. Shen, Journal of Asian Ceramic Societies, 1, 315 (2013).

5. A. Simchi, Materials Science and Engineering: A, 428, 148 (2006).

6. P. Bertrand, F. Bayle, C. Combe, P. Goeuriot and I. Smurov, Applied Surface Science, 254, 989 (2007).

7. J. Yu, M. Chao, D. Li and M. Li, Journal of Power Sources, 226, 334 (2013).

8. J. Zhang, E. J. Liang and X. H. Zhang, Journal of Power Sources, 195, 6758 (2010).

9. M. Lomberg, E. Ruiz-Trejo, G. Offer, J. A. Kilner and N. P. Brandon, ECS Transactions, 57, 1349 (2013).

10. W. G. Bessler, M. Vogler, H. Stormer, D. Gerthsen, A. Utz, A. Weber and E. Ivers-Tiffee, Phys Chem Chem Phys, 12, 13888 (2010).

11. A. Utz, H. Störmer, A. Leonide, A. Weber and E. Ivers-Tiffée, Journal of The Electrochemical Society, 157, B920 (2010).

12. R. Radhakrishnan, A. V. Virkar and S. C. Singhal, Journal of The Electrochemical Society, 152, A210 (2005).

13. A. Bieberle, L. P. Meier and L. J. Gauckler Journal of The Electrochemical Society, 148, A646 (2001).

14. R. Li, J. Liu, Y. Shi, L. Wang and W. Jiang, Int J Adv Manuf Technol, 59, 1025 (2012).

15. M. Islam, T. Purtonen, H. Piili, A. Salminen and O. Nyrhilä, Physics Procedia, 41, 835 (2013).

16. H. J. Niu and I. T. H. Chang, Scripta Materialia, 41, 1229 (1999).

17. J. P. Kruth, L. Froyen, J. Van Vaerenbergh, P. Mercelis, M. Rombouts and B. Lauwers, Journal of Materials Processing Technology, 149, 616 (2004).

18. I. Gibson, D. Rosen and B. Stucker, Additive Manufacturing Technologies. Rapid Prototyping to Direct Digital Manufacturing, Springer US (2010).

19. M. V. Allmen, Laser-beam Interactions with Materials, Springer-Verlag, Berlin (1987). 